This is a preprint of the paper published in Landslides

Accepted citation: Li, S., Peng, C., Wu, W., Wang, S., Chen, X., Chen, J., Zhou, G.G. and Chitneedi, B.K., 2020. Role of baffle shape on debris flow impact in step-pool channel: an SPH study. Landslides, 17, pp.2099-2111.

DOI: $10.1007 / \mathrm{s} 10346-020-01410-\mathrm{w}$

\title{
Role of baffle shape on debris flow impact in step-pools channel: an SPH study
}

\author{
Shuai Li ${ }^{\mathrm{a}}$, Chong Peng ${ }^{\mathrm{b}}$, Wei Wu ${ }^{\mathrm{b}, *}$, Shun Wang ${ }^{\mathrm{b}}$, Xiaoqing Chen ${ }^{\mathrm{a}}$, Jiangang Chen ${ }^{\mathrm{a}}$, Gordon G. D. Zhou ${ }^{\mathrm{a}}$, Bhargav \\ K. Chitneedi ${ }^{\mathrm{c}}$ \\ ${ }^{a}$ Key Laboratory of Mountain Hazards and Earth Surface Process, Institute of Mountain Hazards and Environment, Chinese Academy of Sciences, \\ 610041 Chengdu, China \\ ${ }^{b}$ Institut für Geotechnik, Universität für Bodenkultur, Feistmantelstraße 4, 1180 Vienna, Austria \\ ${ }^{c}$ ESS Engineering Software Steyr GmbH, Berggasse 35, 4400 Steyr, Austria
}

\begin{abstract}
Drainage channels with step-pool system are widely used to control debris flow. The blocking of debris flow often gives rise to local damage at the steps and baffles. Hence, the estimation of impact force of debris flow is crucial for designing step-pools channel. Existing empirical models for impact pressure prediction cannot consider the influence of baffle shape. In this work, a three-dimensional smoothed particle hydrodynamics (SPH) study on the impact behaviour of debris flows in step-pool systems is presented, where debris material is modelled using the regularized Bingham model. The SPH method is first checked using the results from two laboratory tests. Then it is used to investigate the influence of baffle shape and flow density. Numerical results show that the impact pressure at the first baffle highly depends on the baffle shape; however, the largest impact pressure usually occurs at subsequent baffles due to the violent impact induced by jet flows. The peak impact pressure at the first baffle initially grows with increasing flow density; however, it starts to drop as density is beyond a threshold. Based on the numerical results, an empirical relation considering the influence of baffle shape is proposed for better prediction of debris impact pressure.
\end{abstract}

Keywords: Debris flow, Impact pressure, Step-pool systems, Baffle configuration, Smoothed particle hydrodynamics

\section{Introduction}

The step-pool bed configuration, a common geomorphologic phenomenon in high-gradient mountainous terrain (3-30\%), consists of a sequence of steps and pools forming a staircase-like structure in longitudinal profiles (Chin, 1999; Waters and Curran, 2012). This self-organized system can enhance channel bed resistance, slow down mass flows, and effectively dissipate flow energy Maxwell et al. (2001); Wilcox et al. (2011). Inspired by this interesting phenomenon in natural stream flow, similar protection systems with step-pools were introduced to mitigate debris flow Yu et al. (2007); Wang et al. (2012). For instance, Wang et al. Wang et al. (2012) reported an experiment on debris flow on artificial step-pool system. It was found that the step-pool system can result in increased energy dissipation; thus, it reduces the erosion of gully bed and the risk of debris flow outbreak.

Debris-flow drainage channels are important hazard mitigation infrastructure because they are usually constructed in the headwaters of deposit fan, where the life lines are susceptible to debris flow hazards. On the other hand, the analogy between step-pool sequences and energy dissipation structures stimulates the investigation of artificial steppool in drainage channels. A number of studies were carried out to understand the transport processes of debris flow in drainage channel with artificial step-pools Chen et al. (2009, 2015, 2018); Huang et al. (2009); Liu et al. (2016). These experimental studies indicate that the step-pool systems are capable of maximizing flow resistance, reducing velocity, and dissipating the energy of debris flow.

For debris flow drainage channels with step-pools, the stability of steps and baffles is the key factor for the safe operation. Generally, the foundations of steps and baffles in the step-pool system are prone to fail (see Fig. 1) due to the large impact force between debris materials and structures, Li (1997); Fei and Shu (2004). Therefore, the stability

${ }^{*}$ Corresponding author. E-mail: wei.wu@boku.ac.at 
and durability of step-pools under debris flow impact forces should be carefully considered when designing drainage channels.
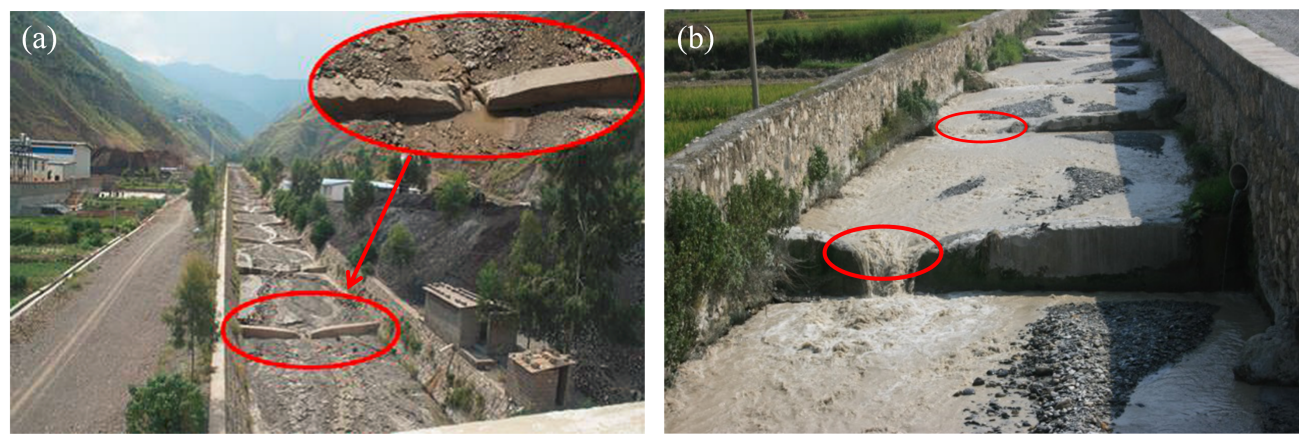

Figure 1: Site examples of broken step-pool in debris-flow drainage channels of (a) Daduo (b) Daqiaohe.

Dynamic impact of debris flow has long been a research subject of considerable interest. Many flume experiments Hübl and Holzinger (2003); Zanuttigh and Lamberti (2006); Armanini et al. (2011); Cui et al. (2015); Vagnon and Segalini (2016); Choi et al. (2017); Song et al. (2018), and some field tests Zhang (1993); Hu et al. (2011); Wendeler et al. (2007) were conducted to study the impact pressure of debris flows. Based on these results, several models were proposed by integrating either hydrostatic or dynamic pressures to estimate the impact force. In these models, the impact pressure of debris flows is assumed to be proportional to the flow depth and the square of flow velocity. Apart from the flow depth and velocity, the impact pressure is also highly dependent on the geometry of structures Shieh et al. (2008). However, little attention has been paid to the impact pressure in step-pool systems with different baffle shapes. This may be attributed to the fact that conventional approaches like physical modelling and field testing are either time-consuming or costly. Furthermore, in physical modelling it is usually difficult to get all the desired flow properties. On the other hand, field testing involves high degree of uncertainty, and is sometimes dangerous.

Alternatively, numerical simulation can be applied to investigate debris flow impact. Traditionally, depth-integration methods are widely used in debris flow modelling owing to their relative simplicity and efficiency Hutter et al. (1994); Iverson and Denlinger (2001). However, it is difficult to obtain accurate impact force using depth integration methods, especially for problems with complex flow dynamics and violent free surfaces involving jumps and fragmentations. On the other hand, three-dimensional simulations, which consider the complete mass and momentum conservations, can provide detailed results on impact forces and flow dynamics. Nevertheless, not all numerical methods are suitable for this task. Traditional grid-based methods such as finite element method (FEM) or finite volume method (FVM) need to track the violent free-surface using additional techniques Larese et al. (2008), which usually involve complex formulation and implementation, increased computational cost, and numerical error Lv et al. (2011).

Recently, particle-based methods are developed for problems with free-surface flows and large deformation, e.g. Discrete Element Method (DEM) Leonardi et al. (2019); Calvetti et al. (2019), Material Point Method (MPM) Fern and Soga (2016), Smoothed Particle Hydrodynamics (SPH) Neto and Borja (2018), and Particle Finite Element Method Li et al. (2018). Among them, smoothed particle hydrodynamics (SPH) is well established and has a long application history in computational fluid dynamics Monaghan (1994) and solid mechanics Monaghan (2000). Owing to its meshless and Lagrangian nature, SPH is suitable for problems with violent free-surface and large deformation. Consequently, in the past years SPH was used in landslide and debris flow modelling Chambon et al. (2011); Huang et al. (2012); Dai et al. (2017); Peng et al. (2016); Wang et al. (2016); Pastor et al. (2018). The results indicate that the $\mathrm{SPH}$ is robust and reliable in modelling the fundamental dynamic behaviour of mass flows. Therefore, SPH is adapted in this work to investigate the influence of baffle shape on the impact pressure in step-pool systems. To accelerate the SPH computations, acceleration based on Graphic Processing Unit (GPU) is utilized.

The remaining part of the paper is organized as follows. First, the SPH method with a regularized Bingham model is introduced. Then, two flume tests on triangular and square baffles with fixed densities are carried out and the results are used as benchmarks to check the performance of the SPH model. With the calibrated SPH model, the impact force on three baffle shapes, i.e. square, triangular, and trapezoidal, are investigated under varying flow densities. Finally, the numerical results are compared with empirical models from literature. A new relation considering the influence 
of baffle shape is proposed to estimate the impact pressure.

\section{SPH with a regularized Bingham model}

\subsection{Governing equations and constitutive model}

The governing equations for debris flows consisting of the classical (Navier-Stokes) mass and momentum conservation equations are as follows

$$
\begin{gathered}
\frac{\mathrm{d} \rho}{\mathrm{d} t}=-\rho \nabla \cdot \boldsymbol{v} \\
\frac{\mathrm{d} \boldsymbol{v}}{\mathrm{d} t}=\frac{1}{\rho} \nabla \cdot \boldsymbol{\sigma}+\boldsymbol{g}
\end{gathered}
$$

where $\mathrm{d}(\cdot) / \mathrm{d} t$ represents the material time derivative, $\nabla$ is the gradient operator and $\cdot$ denotes the inner product. $\rho$ and $v$ are the flow density and velocity. $\sigma$ and $\boldsymbol{g}$ denote Cauchy stress tensor and body force, respectively. The stress tensor $\sigma$ can be decomposed into a pressure and a shear stress tensor:

$$
\sigma=p \boldsymbol{I}+\boldsymbol{\tau}
$$

where $\boldsymbol{I}$ is an identity matrix.

In this work, debris materials are simulated as a weakly-incompressible non-Newtonian fluid. This assumption allows us to compute the pressure directly from density using an equation of state as:

$$
p=c^{2}\left(\rho-\rho_{0}\right)
$$

where $c$ is the speed of sound taken as a numerical constant, $\rho_{0}$ is the reference density when there is no pressure. More complex equations of state are also found in SPH computations, but the results are essentially identical Chambon et al. (2011); Marrone et al. (2011).

In this study, debris materials are modeled using the Bingham model, which is widely used in debris flow modelling Dai et al. (2014); Wang et al. (2016); Lin et al. (2019). The Bingham model is suitable for debris materials with considerable fine particles, where the flow exhibit strong viscous behaviors. The debris materials employed in the experimental tests are such type of material; thus, the Bingham model is adopted. The model is characterized by the existence of a threshold termed yield stress $\tau_{0}$. Only when the stress state reaches the yield condition the material starts to flow. Beyond the yield stress, the material behaves like a Newtonian flow with a constant viscosity $\eta_{0}$. In general, the shear stress tensor is computed as:

$$
\tau=2 \eta_{a p p} \dot{\boldsymbol{\varepsilon}}
$$

where $\eta_{a p p}$ is the apparent viscosity, and $\dot{\boldsymbol{\varepsilon}}$ is the strain rate tensor defined as $\dot{\boldsymbol{\varepsilon}}=\left[\nabla \boldsymbol{v}+(\nabla \boldsymbol{v})^{\mathrm{T}}\right] / 2$. In Bingham model, the apparent viscosity has the following form:

$$
\eta_{a p p}=\eta_{0}+\tau_{0} / \dot{\gamma}
$$

where $\dot{\gamma}$ is defined as $\dot{\gamma}=\sqrt{2 \dot{\varepsilon}: \dot{\varepsilon}}$. It is found that the above model is singular in the static state where $\dot{\gamma}=0$. Therefore, we employ the regularized model proposed by Zhu et al. Zhu et al. (2005), which has the following form:

$$
\eta_{a p p}=\eta_{0}+\frac{\tau_{0}}{\dot{\gamma}}\left(1-e^{-m \dot{\gamma}}\right)
$$

where $m$ is a regularization parameter which has the dimension of time. With this regularization, when the strain rate tends to zero, the viscosity has a limit $\eta_{0}+\tau_{0} \mathrm{~m}$. It is found that the regularized Bingham model is closer to the original Bingham model if a larger $m$ is used. However, a very large $m$ leads to extremely high apparent viscosity in the quasi-static flow region, which results in very small time step in the SPH method and prohibitive computational cost. In numerical experiments, we found that simulations using $m=100 \mathrm{~s}$ and $m=1000 \mathrm{~s}$ give almost identical flow dynamics. Therefore, $m=100 \mathrm{~s}$ is used throughout the work. 


\subsection{SPH formulations}

In SPH, the computational domain is partitioned using particles which can be viewed as material elements carrying field variables and moving with the material. The carried variables usually include mass, density, stresses, velocity components, and other needed field variables. The governing equations can be solved by tracking the motions of the particles and changes of the carried variables.

The essential part of SPH is to approximate a function $f(\boldsymbol{x})$ and its gradient $\nabla f(\boldsymbol{x})$ through the discrete values $f$ in the vicinity of $\boldsymbol{x}$ using a weighting function

$$
\begin{gathered}
f_{i}=\sum_{j=1}^{n} \frac{m_{j}}{\rho_{j}} f_{j} W_{i j} \\
\nabla f_{i}=\sum_{j=1}^{n} \frac{m_{j}}{\rho_{j}} f_{j} \nabla_{i} W_{i j}
\end{gathered}
$$

where $f_{i}$ and $\nabla f_{i}$ are the approximated function and gradient at the position $\boldsymbol{x}_{i}$, whereas $f_{j}, m_{j}$, and $\rho_{j}$ are the function value, mass, and density at the position $\boldsymbol{x}_{j}$, respectively. $W_{i j}=W\left(\boldsymbol{x}_{i}-\boldsymbol{x}_{j}, h\right)$ is the weighting function called smoothing kernel. The weighting function depends on the scalar distance between the two positions $\boldsymbol{x}_{i}$ and $\boldsymbol{x}_{j}$ and the constant $h$, called smoothing length. The soothing length $h$ determines the size of the weighting function, and $n$ is the number of particles in the influence area of the kernel function. Various smoothing kernels are employed in SPH computation. Here the C2 Wendland kernel function Wendland (1995) is used.

By using the SPH kernel approximation, the governing equations can be rewritten as the following discretized form

$$
\begin{gathered}
\frac{\mathrm{d} \rho_{i}}{\mathrm{~d} t}=\sum_{j=1}^{n} m_{j}\left(\boldsymbol{v}_{i}-\boldsymbol{v}_{j}\right) \cdot \nabla_{i} W_{i j}+\delta h c \sum_{j=1}^{n} \frac{m_{j}}{\rho_{j}} \boldsymbol{\psi}_{i j} \cdot \nabla_{i} W_{i j} \\
\frac{\mathrm{d} \boldsymbol{v}_{i}}{\mathrm{~d} t}=\sum_{j=1}^{n} m_{j}\left(\frac{p_{i}}{\rho_{i}^{2}}+\frac{p_{j}}{\rho_{j}^{2}}+\Pi_{i j}\right)+\sum_{j=1}^{n} \frac{2 m_{j}\left(\eta_{i}+\eta_{j}\right) \boldsymbol{r}_{i j} \cdot \nabla_{i} W_{i j}}{\rho_{i} \rho_{j}\left(\boldsymbol{r}_{i j}^{2}+0.01 h^{2}\right)} \boldsymbol{v}_{i j}+\boldsymbol{g}_{i}
\end{gathered}
$$

Eq. (10) is the SPH discretized form of the mass conservation equation. It is well-known that the classical SPH method suffers from pressure oscillations. To increase the accuracy of impact force computation, a numerical diffusion term called $\delta$-SPH is employed with $\delta=0.1$ Marrone et al. (2011). The numerical diffusion term smooths out the oscillations and results in better pressure results.

The first and second terms in the discretized momentum conservation equation (11) denote the contribution from pressure gradient and shear stress gradient, respectively. The discretization of the shear stress gradient follows the form employed in Szewc Szewc (2017). For details of the derivation, interested readers can refer to Szewc Szewc (2017) and the references therein. Unlike the form used in Chambon Chambon et al. (2011), the above momentum equation does not require the storage of shear stress tensor and strain rate tensor. Compared with SPH simulation of Newtonian fluids, only one additional scalar (the apparent viscosity $\eta_{a p p}$ ) at each particle is needed, giving rise to reduced memory consumption.

In the discretization of the pressure gradient, the stabilization term $\Pi_{i j}=\Pi_{i j}^{v i s}+\Pi_{i j}^{p}$ consists of the corrections by artificial viscosity $\Pi_{i j}^{v i s}$ and artificial pressure $\Pi_{i j}^{p}$. The artificial viscosity is used to stabilize the simulation and damp out numerical oscillations, whereas the artificial pressure is employed to cure the tensile instability widely observed in non-Newtonian flows with high viscosity Xu et al. (2013). The employed artificial viscosity and artificial pressure have the same forms as those proposed by Monaghan in his classical papers Monaghan $(1994,2000)$. For the sake of brevity, the detailed forms are omitted here. The coefficients used in the simulations are: $\alpha_{1}=0.1$ and $\alpha_{2}=0.0$ for artificial viscosity, and $b=0.2$ for artificial pressure.

\subsection{Numerical implementation}

The treatment of solid boundaries in SPH need to fulfil two requirements, the first is to prevent particle penetration, and the second is to correct the SPH approximation in incomplete support domains due to the intersection with solid boundaries. In this study, the non-slip solid wall boundary is implemented using the boundary particle method Adami 
et al. (2012), where the solid boundary is represented by certain layers of SPH particles. The boundary particles are either fixed in position or moved according to prescribed velocities. The boundary particles participate in SPH computations like normal material particles. However, the field variables on the boundary particles are not computed by evolving the governing equations, but are extrapolated from the material domain. Specially, except the common variables such as pressure, density, and velocity, in simulations of debris material, the apparent viscosity also needs to be extrapolated to the boundary particles to correctly evaluate the discrete governing equations.

In the SPH model, the system of equations consisting of Eq. (10) and (11) is solved by explicit time integration using the Predictor-Corrector scheme Monaghan (1994). The time step of the Predictor-Corrector scheme is controlled by the CFL condition Chambon et al. (2011)

$$
\Delta t=\chi \min \left(\frac{h}{c}, \frac{h}{\max \left\|\boldsymbol{a}_{i}\right\|}, h^{2} \min \frac{\rho_{i}}{\eta_{a p p, i}}\right)
$$

where $\boldsymbol{a}_{i}=\mathrm{d} \boldsymbol{v}_{i} / \mathrm{d} t$ is the particle acceleration, $\chi$ is the Courant-Friedrichs-Lewy (CFL) coefficient usually in the range of 0.05-0.2. Compared to simulations with low viscosity Newtonian fluid like water, simulations with debris material have additional time restriction induced by viscosity.

Generally, SPH has high computational cost due to large amount of particle interaction, frequent neighbor search, and small time step in numerical integration. In this work, the three-dimensional chute flow results in numerical model of millions of SPH particles, which is prohibitive for serial computing. Parallel computing is therefore required. Recently, it becomes popular to use graphic processing units (GPUs) to perform parallel computing in SPH Hérault et al. (2011); Crespo et al. (2015); Xia and Liang (2016); Peng et al. (2019); Zhan et al. (2019); Chen et al. (2020). The GPU parallelization allows SPH simulations with millions to dozens of millions of particles to be carried out using a single GPU device installed on a desktop.

In this work, the regularized Bingham model and the related algorithms are implemented based on the GPUaccelerated open-source code LOQUAT Peng et al. (2019). In the GPU implementation, the computational procedure of debris flow follows the same process as that for pure water and geomaterials modelled using soil constitutive models Peng et al. (2019). However, in each step one additional round of particle interaction is required to evaluate the shear strain rate, which is used to compute the apparent viscosity using the regularized Bingham model.

\section{Experimental set-up and tests}

To check the performance of the SPH method in modelling impact pressure with varying baffle shape and flow density, experimental tests are carried out to provide validation data. To this end, an artificial step-pool system is built in a drainage channel. The step-pool system consists of a sequence of steps and baffles, which in lateral profile resembles a staircase. The configurations and dimensions of the channel are shown in Fig. 2. The test system has a tilting flume (with an inclination of $\theta=8.5^{\circ}$ ) of $0.45 \mathrm{~m}$ width, $0.40 \mathrm{~m}$ depth, and $6.0 \mathrm{~m}$ length. A storage tank and a tailing pool is placed at the top and end of the flume. Five artificial step-pool sequences are installed in the debris flow drainage channel. Two shapes of the baffle, i.e. square and triangle, are considered in the tests. A third baffle shape, namely trapezoid, is not considered in the tests but will be investigated in numerical simulations. In the three configurations, the inclined angles $(\beta)$ of baffles respect to incoming flows are $90^{\circ}, 45^{\circ}$, and $63^{\circ}$ for square, triangle, and trapezoid shapes, respectively (Fig. 2). For each step-pool unit, two pressure sensors are installed at the surfaces of the baffle and the step. From upstream to downstream, the sensor numbers are in order as Pa1 - Pa5 for baffles and $\mathrm{Pb} 1$ - $\mathrm{Pb} 5$ for steps. Additionally, laser sensors used for measuring the height of free-surface of flow are located at 1-1 and 2-2 sections, near the mounted start and end of the flume, respectively. Video cameras are used to record the flow process, particularly the impact of the debris flow onto the step-pools.

In a test, the prepared debris material with designed solid volume fraction is first loaded in the storage tank. Then, the bottom gate of the storage tank is released to allow the debris material flow into the flume. The material flows down the flume and impacts with the baffles, and is finally collected by the tailing tank. The test debris material is a mixture of water and a natural soil from deposit fun of Jiangjia Ravine in China. The grain size of the sediment extends from 0.001 to $20 \mathrm{~mm}$, with the intermediate grain size $d_{50}=5.1 \mathrm{~mm}$. Five types of debris materials (M1 - M5) are prepared with varying solid volume fraction. The material properties including density, yield stress, and dynamic viscosity are listed in Table 1 . The yield stresses and dynamic viscosities are measured using a rheometer. 

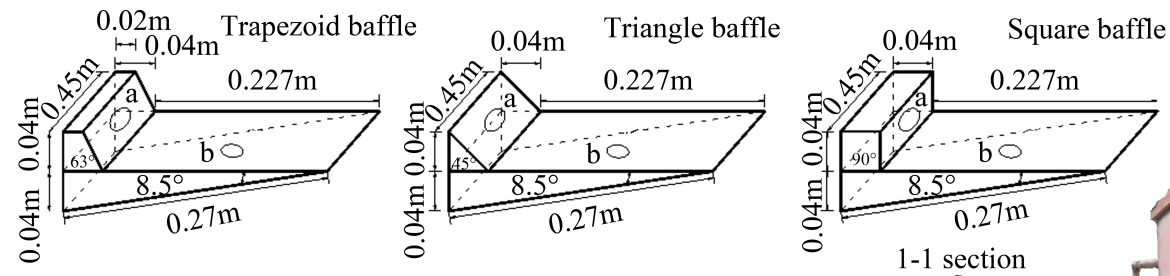

a: Impact stress sensor on baffle
b: Impact stress sensor on step
c: Video Camera
d: Laser Mud Level Sensor

\section{2-2 section}

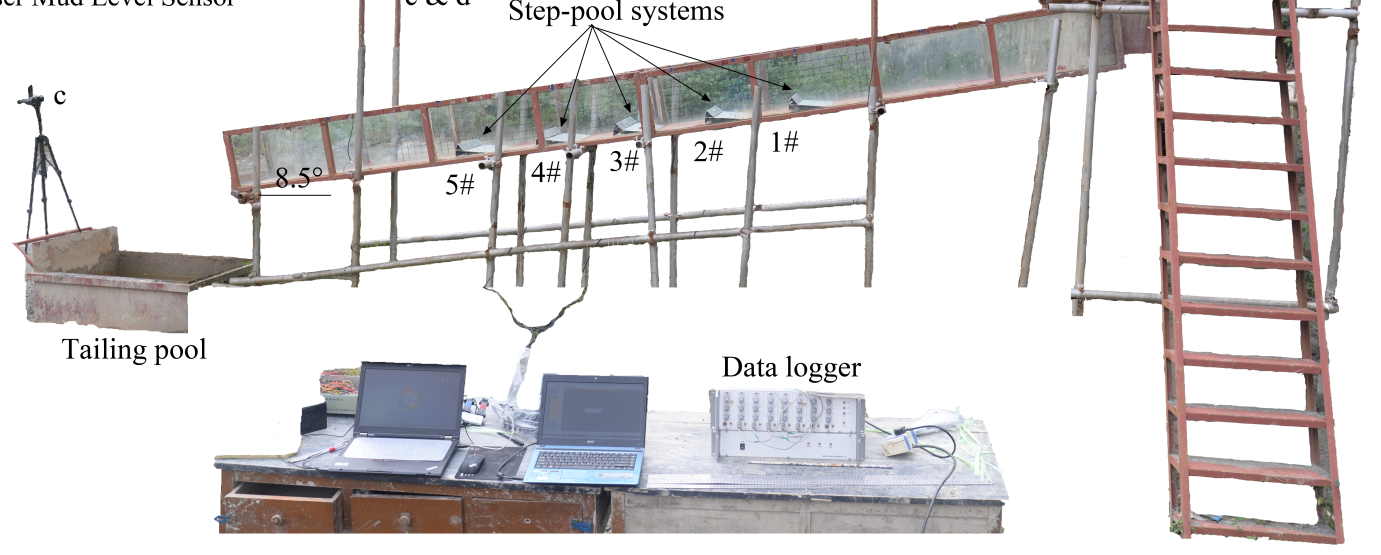

Figure 2: Overview of the model equipment, baffle configurations and pressure measurement points.

Table 1. Characteristic parameters of debris materials

\begin{tabular}{ccccc}
\hline Type & Density $\left(\mathrm{kg} / \mathrm{m}^{3}\right)$ & Solid volume fraction $(-)$ & Yield stress $(\mathrm{Pa})$ & Dynamic viscosity $(\mathrm{Pa} \cdot \mathrm{s})$ \\
\hline M1 & 1828 & 0.48 & 14.00 & 0.0672 \\
M2 & 1959 & 0.55 & 25.66 & 0.1231 \\
M3 & 2129 & 0.65 & 49.57 & 0.2379 \\
M4 & 2200 & 0.69 & 78.48 & 0.3767 \\
M5 & 2259 & 0.72 & 103.15 & 0.4951 \\
\hline
\end{tabular}

Two debris flow impact tests (M2 with triangle baffles and M3 with square baffles) are performed. These two tests serve as benchmarks to calibrate the developed SPH model. The other three types of material (M1, M4, and M5) and one type of baffle shape (trapezoid) are considered in numerical simulations.

\section{Validation cases}

In order to check the performance of the SPH model in simulating the flow dynamics and flow-baffle interaction, two numerical simulations with the same boundary conditions and materials properties as those in the two tests (M2 with triangle baffles and M3 with square baffles) are carried out. The flume, storage tank, and the debris material in the tank are discretized using SPH particles of $\Delta r=0.004 \mathrm{~m}$. The symbol $\Delta r$ is the particle size in SPH, which is a numerical resolution like the element size in finite element method. The particle numbers used in the simulations, including the simulations for the trapezoid configuration considered in the next section, are given in Table 2 .

The variables used in the comparison are the change of flow depth at the section 1-1 and the impact pressure on the surface of the first baffle. The results of flow depth from the numerical simulations and tests are shown in Fig. 3. Generally, good agreement between numerical and experimental results is achieved. The numerical prediction of the arriving time and the change of depth before $t=1 \mathrm{~s}$ are very accurately captured by the SPH model in both two cases. However, the final deposition depths are slightly overestimated. This discrepancy might be caused by the 
Table 2. Summarizes the configurations and the number of particles for all the simulations

\begin{tabular}{cccc}
\hline Configurations & Total particles & Material particles & Boundary particles \\
\hline Square & $3,192,089$ & $2,094,722$ & $1,097,367$ \\
Triangle & $3,149,288$ & $2,094,722$ & $1,054,566$ \\
Trapezoid & $3,183,311$ & $2,094,722$ & $1,088,589$ \\
\hline
\end{tabular}

use of the Bingham model, which is rather simple hence cannot describe detailed deposition behaviors of the debris materials. Nevertheless, the general trends of the change of flow depth are well simulated. Particularly, the SPH model successfully captures the abrupt changes of depth induced by unsteady surge flows.
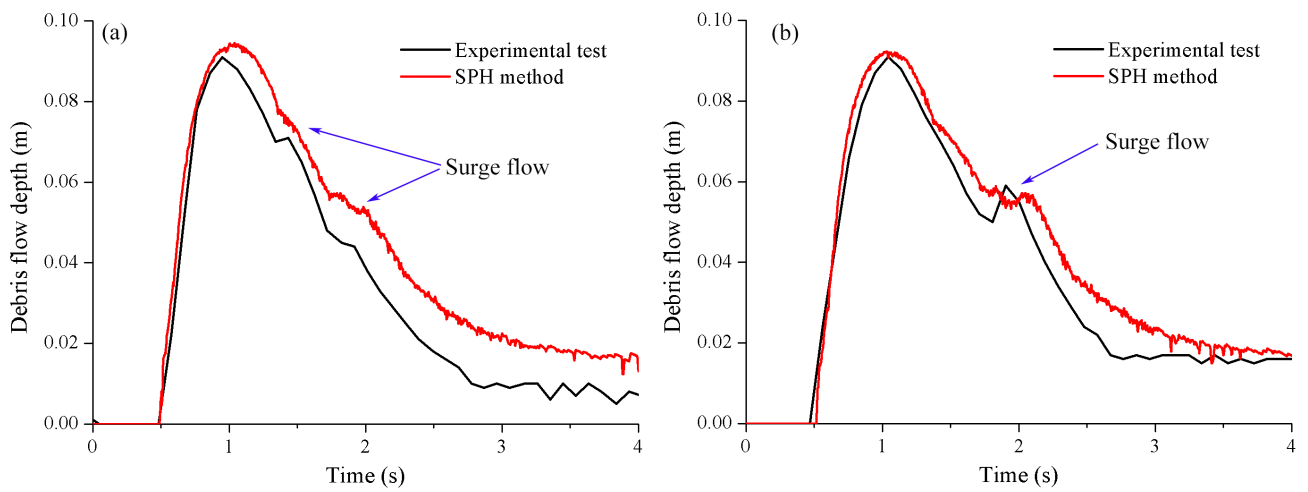

Figure 3: Comparison of the debris flow depth at the $1-1$ section between the experimental results and SPH method: (a) $\rho=1959 \mathrm{~kg} / \mathrm{m}^{3}$ with triangle baffles, (b) $\rho=2129 \mathrm{~kg} / \mathrm{m}^{3}$ with square baffles.

Furthermore, the impact pressures on the surfaces of the first baffle are shown in Fig. 4. The peak impact pressures in numerical simulations and experimental tests both correspond to the impact between the first baffle and the flow front, although some fluctuations present in the experimental results. The numerical and experimental results of impact pressure agree well with each other in terms of general trend, peak pressure, and arriving time. Through the comparison between the numerical simulations and tests in the two cases, it is found that the SPH model with the regularized Bingham model can capture the salient features of the debris flow in step-pool system with varying material properties and baffle geometries.
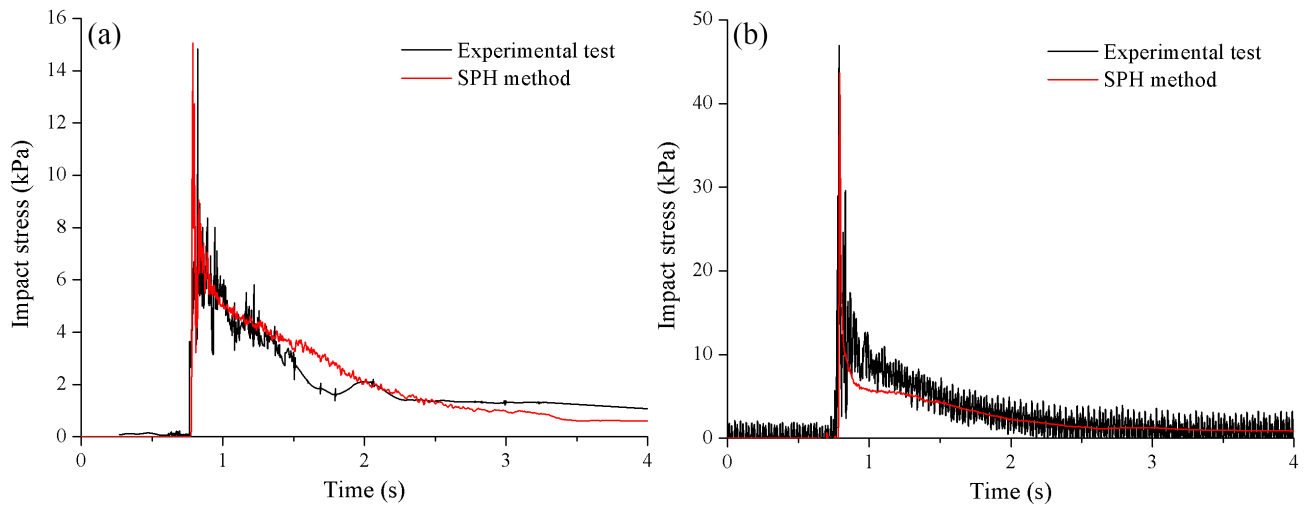

Figure 4: Comparison of the impact stress on the 1 baffle between the experimental results and SPH method; (a) $\rho=1959 \mathrm{~kg} / \mathrm{m}^{3}$ with triangle baffles, (b) $\rho=2129 \mathrm{~kg} / \mathrm{m}^{3}$ with square baffles. 


\section{Simulations with varying baffle shape and flow density}

In total, we performed 15 simulations for three baffle shapes shown in Fig. 2. In all simulations the channel geometry and the material volume are the same, the only differences are the baffle shape and material property. For each baffle shape, five simulations are performed with the five sets (M1 - M5) of material properties listed in Table 1. The numerical resolution is $\Delta r=0.004 \mathrm{~m}$, and the resultant numbers of particle in the simulations are listed in Table 2.
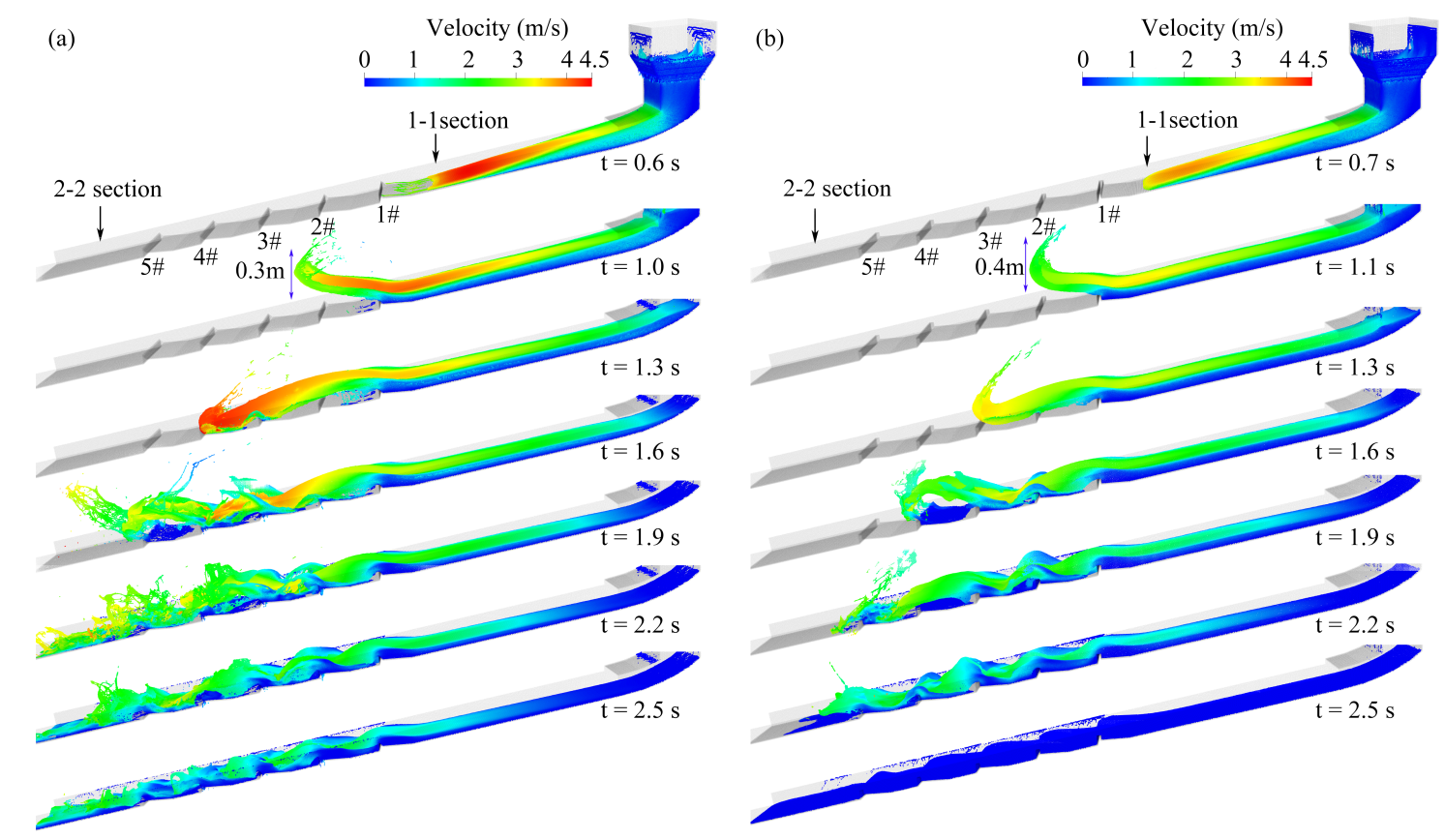

Figure 5: Snapshots of the profiles of debris flow as well as the velocity field at different time with (a) $\rho=1959 \mathrm{~kg} / \mathrm{m}^{3}$ with triangle baffles, (b) $\rho=2129 \mathrm{~kg} / \mathrm{m}^{3}$ with square baffles.

\subsection{Flow process}

The simulated flow processes in two cases, i.e., $\rho=1959 \mathrm{~kg} / \mathrm{m}^{3}$ with triangle baffles, and $\rho=2129 \mathrm{~kg} / \mathrm{m}^{3}$ with square baffles, are shown in Fig. 5. The time measurement starts when the debris material is released from the storage tank. The times when the flow front reaches section $1-1$ are $0.6 \mathrm{~s}$ and $0.7 \mathrm{~s}$ for the cases with densities of $1959 \mathrm{~kg} / \mathrm{m}^{3}$ and $2129 \mathrm{~kg} / \mathrm{m}^{3}$, respectively. It is also observed that the debris material with a larger density has a lower velocity after travelling the same distance. This is attributed to the difference in material properties. Usually, a debris material with a high density exhibits more evident viscous behaviour, thereby leading to lower velocity and longer moving time. It is also observed that the debris flow sharply jumps up the first baffle when its front reaches the 1\# baffle. The maximum jump height in the case with square baffle is approximately $0.4 \mathrm{~m}$, which is larger than that observed in the case with triangle baffle (approximately $0.3 \mathrm{~m}$ ). The result implies that the square baffle gives rise to more violent change of the flow in comparison to the triangle baffle with inclined angle of $45^{\circ}$.

Due to the uplift effect at the 1\# baffle, for the step-pool with square baffles, the debris flow jumps over the 2\# baffle and hits the $3 \#$ baffle $(t=1.3 \mathrm{~s}$ in Fig. 5b). For the triangle baffle step-pool, however, the debris flow can jump over the $2 \#$ and $3 \#$ baffles $(t=1.3 \mathrm{~s}$ in Fig. 5a), since the triangle baffle imposes less resistance to the debris material in the horizontal direction. The downstream baffles again lift the debris flow, thereby causing a flow-wave in the drainage channel until the material in the channel eventually stops flowing. The step-pool system causes violent free-surface flows with strong fragmentations and impacts between debris materials and baffles.

The flow dynamics in the remaining 13 simulations are similar to that shown in Fig. 5. However, in each simulation, the arriving times of the debris fronts at the baffles may be different.This is because the distance, which the jet flow travels in the air after the first impact, varies from case to case. The arriving times at different baffles in all the 

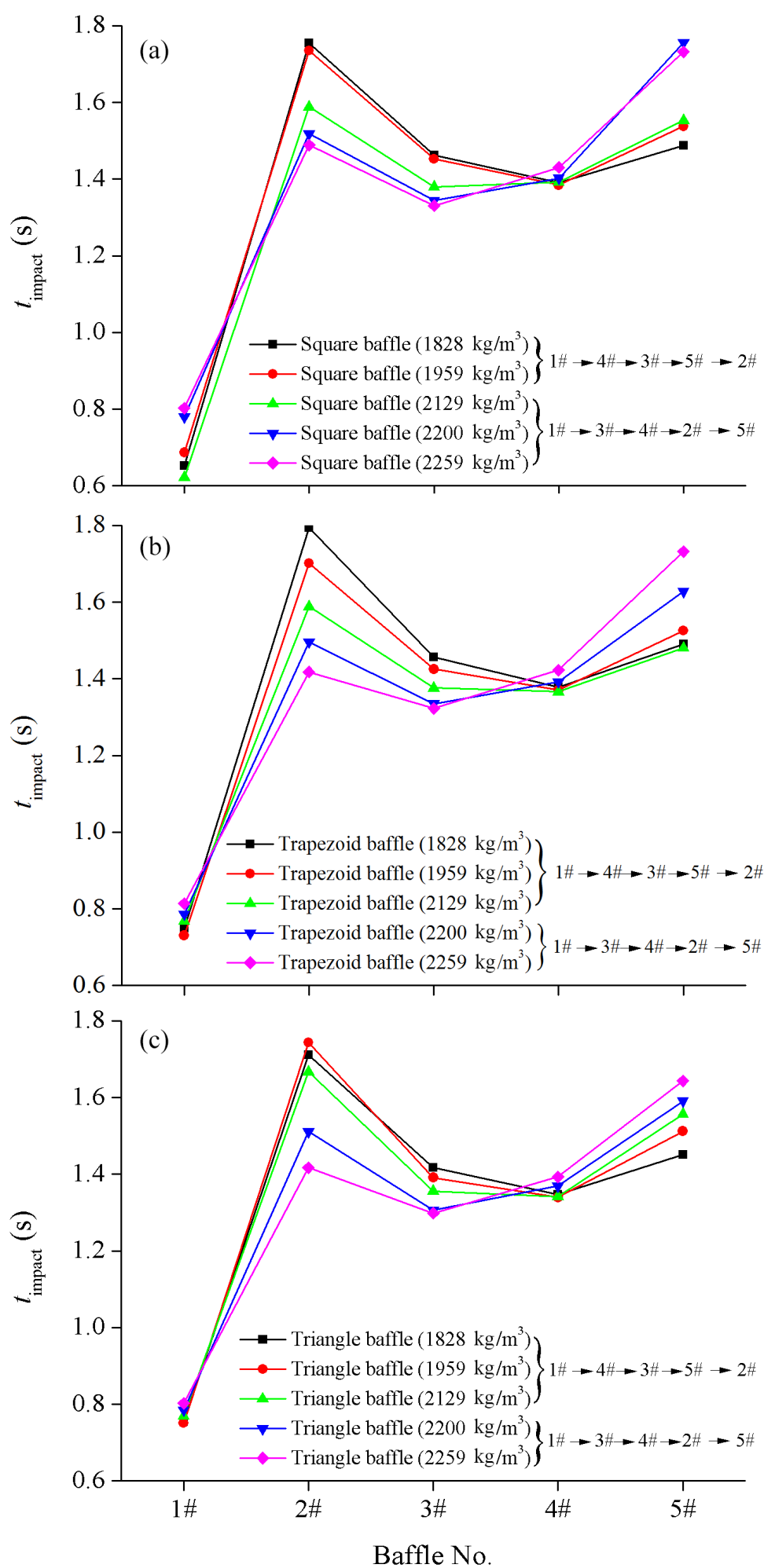

Figure 6: Time for the different density of debris flows impact the (a) square baffles, (b) trapezoid baffles, and (c) triangle baffles. 


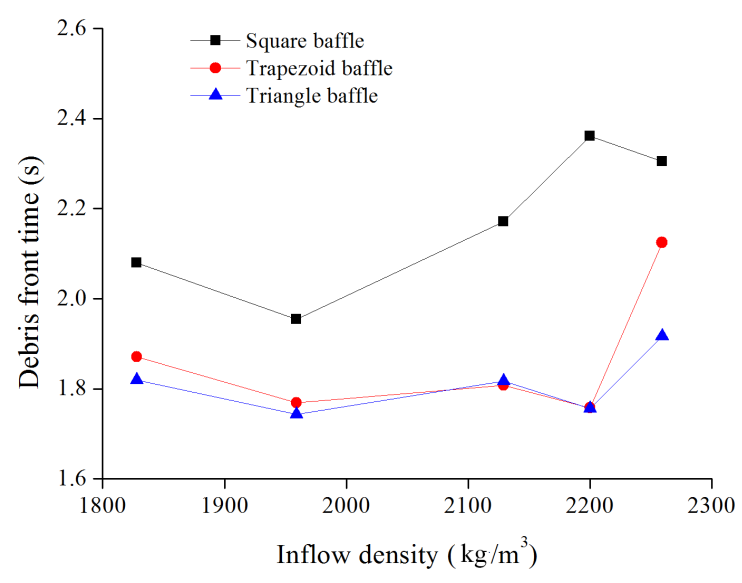

Figure 7: Arriving time at the section 2-2.

simulations are shown in Fig. 6. It can be seen that regardless of the baffle shape, the arriving times of the debris flow is inconsistent with the order of the baffles. This inconsistency is due to that the jet flow, caused by the impact at the first baffle, skips some baffles during flying.

The travelling distance of the jet flow depends on the material properties and baffle shape. For flows with low densities $\left(\rho=1828 \mathrm{~kg} / \mathrm{m}^{3}\right.$ and $\left.1959 \mathrm{~kg} / \mathrm{m}^{3}\right)$, the $2 \#$ baffle is always the last one to get the impact. This is because materials with low density have high fluidity; thus, the flows always jump over the $2 \#$ and $3 \#$ baffles and hit the $4 \#$ baffle directly. As time passes, the velocity of the incoming flow at the 1 \# baffle becomes lower, resulting in shorter travelling distance after impact. Thus, the impact spot gradually moves upstream, first reaches the $3 \#$ baffle, and finally get to the $2 \#$ baffle. Therefore, for flows with low density, the sequence of $t_{\text {impact }}$ is $1 \# \rightarrow 4 \# \rightarrow 3 \# \rightarrow 5 \# \rightarrow 2 \#$. For flows with high densities $\left(\rho=2200 \mathrm{~kg} / \mathrm{m}^{3}\right.$ and $\left.2259 \mathrm{~kg} / \mathrm{m}^{3}\right)$, the trend is similar. However, these dense debris materials have higher viscosity, which results in lower impact velocity at the $1 \#$ baffle and shorter travelling distance of the jet flow. Therefore, the jet flows with these two densities first hit the $3 \#$ baffle. The flow overtops the $3 \#$ baffle and reaches the $4 \#$ baffle. Afterwards, the hit spot of the jet flow moves upstream and hit the $2 \#$ baffle. The sequence of $t_{\text {impact }}$ is therefore $1 \# \rightarrow 3 \# \rightarrow 4 \# \rightarrow 2 \# \rightarrow 5 \#$.

The influence of baffle shape can be clearly observed in the cases with the intermediate density $\rho=2129 \mathrm{~kg} / \mathrm{m}^{3}$. In the simulations with triangle and trapezoid baffles, the jet flows hit the $4 \#$ baffle, whereas in the simulation with square baffles, the jet flows only reaches the $3 \#$ baffle, indicating a shorter flying distance. This is unsurprising, as the inclined surfaces in triangle and trapezoid baffles impose less restriction to the flow in the horizontal direction.

The time at which the flows reach the end of the channel (Section 2-2 in Fig. 2) can be employed to compare the overall flow resistance in the step-pool systems. As shown in Fig. 7, generally, the flows in the channel with square baffles take longer time to arrive at the end of the channel. As the inclination of the baffle surface decreases from $90^{\circ}$ to $45^{\circ}$, the arriving time becomes smaller, indicating lower system resistance and energy dissipation. However, it is also observed sometimes flows in the channel with triangle baffles take longer time to reach the last baffle than those in the channel with trapezoid baffles. This is against our intuition, because it is easy to imagine, that with flatter surface the baffles impose less resistance to the flows, which should result in shorter arriving time. However, the flow dynamics in the step-pool system is very complex due to the jet flow and chaotic impact. The overall resistance not only depends on the baffle shape, but also on the arrangement of the step-pool system, as well as the velocity of the incoming flow, which determines the impact spot of the jet flow. Therefore, it is not always true that baffles with larger inclination can give rise to higher overall resistance.

From Fig. 7, we can further observe that generally, within the simulations using the same baffle shape, a flow with higher density takes a longer time to reach the last baffle. This is because materials with higher density have higher viscosity. However, again, this dependency is rather weak. In many cases, the flows with higher densities arrive at the channel end in a shorter time than those with lower densities. This observation indicates the complexity of flows in step-pool system. The total energy dissipation in the step-pool system highly depends on the material properties, the baffle shape, and the actual configuration of the step-pool system. 


\subsection{Impact force}

The critical factor that triggers the failure of step-pools drainage channel is the peak impact pressure at the barriers and steps. Therefore, the peak impact pressure is adopted as the key quantity to analyse the performance of baffles with different geometries
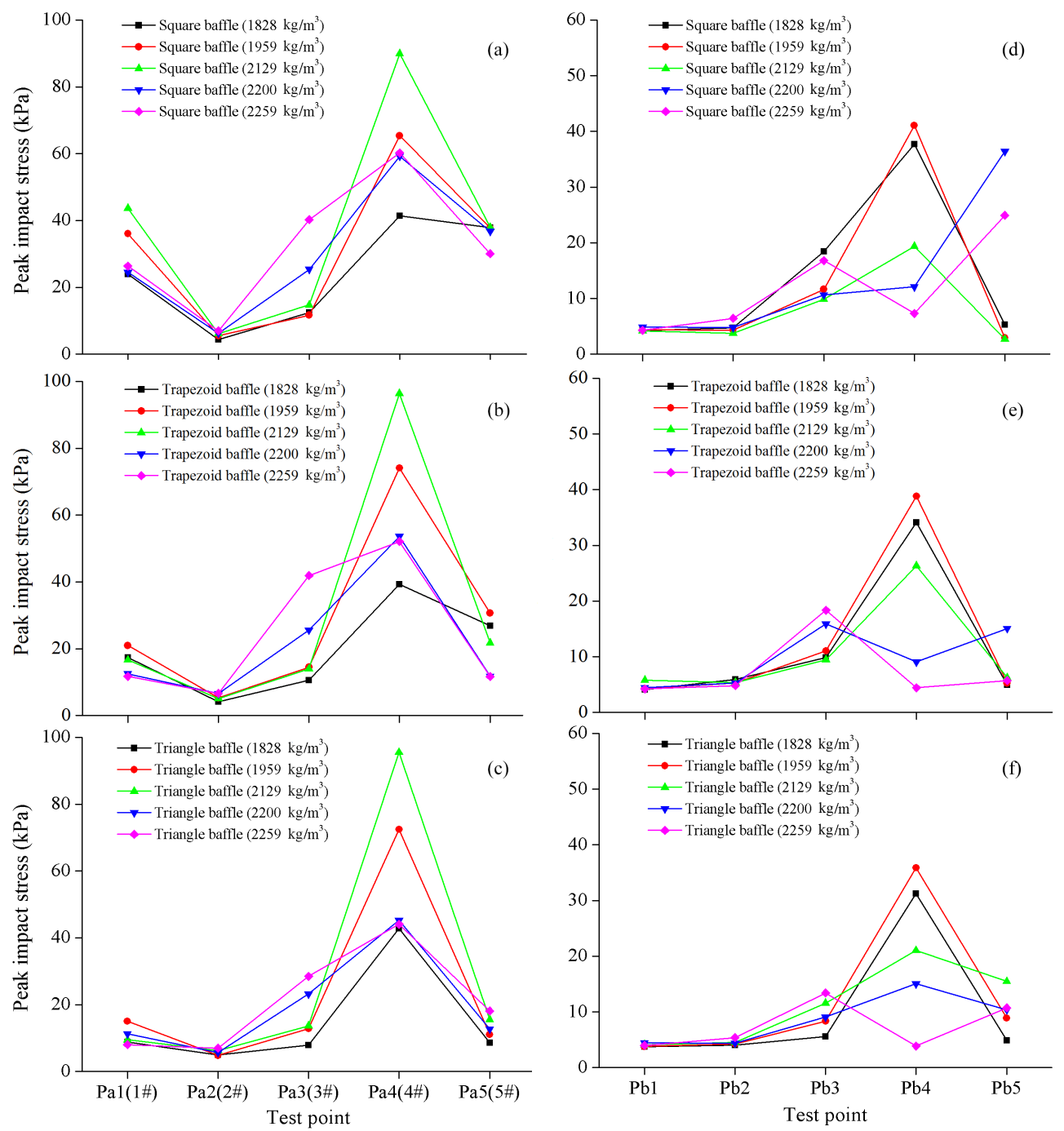

Figure 8: Peak impact stress on the (a) square baffles, (b) trapezoid baffles, (c) triangle baffles; and on the step of (d) square-baffle flume, (e) trapezoid-baffle flume, (f) triangle-baffle flume.

As shown in Fig. 2, pressures on the surfaces of baffles and steps are measured. The results of peak pressures are given in Fig. 8. Generally, the peak impact stresses on each baffle and at the step are different. The largest value of the peak impact stress on baffle surfaces is observed at the $4 \#$ baffle. This is because in all cases, either the whole jet flow, or the most part of it hits directly on the $4 \#$ baffle, giving rise to large impact pressure. For most of the cases, the pressure measured at the baffles is larger than that from the steps. Therefore, the critical part of the step-pool system is the baffles. Special attention should be paid to the stability of baffles when designing step-pool channels.

The influence of the baffle shape on impact force can be clearly observed from the peak pressure at the $1 \#$ baffle. It is found that the high peak pressures are measured at square baffles, while low values are obtained at triangle baffles. For the three type of baffles, the ranges of peak pressure are $24-44 \mathrm{kPa}, 12-21 \mathrm{kPa}$, and $8-15 \mathrm{kPa}$ for square, , and triangle baffles, respectively. This is because the inclinations of the baffle surfaces are $90^{\circ}, 63^{\circ}$, and 
$45^{\circ}$, respectively. A small change in the surface inclination results in a notable difference in the impact pressure. The results indicate that under similar incoming flow conditions (flow direction, velocity, and depth), the impact pressure is highly dependent on the inclination of baffle surface. The relation between impact pressure and inclination of baffle surface at the first baffle will be further discussed in Section 6. For pressures at other baffles, no clear dependence between peak pressure and baffle inclination can be observed. This is again caused by the jet flow and the subsequent complex flow dynamics. The incoming flow conditions at these baffles are not as uniform as those at the first baffle. The flow direction, velocity, and depth have high degree of uncertainties; thus, it is difficult to have clear trends at these baffles.

It is worth noting that in all the cases the peak pressure does not occur at the first baffle. The baffles hit directly by jet flows usually have much larger peak force. This is a critical observation, as in engineering practices the impact pressure is usually estimated using the velocity and density of incoming flows. This estimated pressure only corresponds with the impact pressure at the first baffle. If jet flows happen in the step-pool system, it is very likely some baffles will be subjected to pressures much larger than the estimated value at the first baffle due to the violent impact between baffles and jet flows.

The impact pressure also highly depends on the density of flow. Assuming that all other material properties are the same, a flow with higher density has larger kinetic energy after travelling the same distance, giving rise to higher impact pressure. However, dense flows usually have high viscosities, resulting in lower end speed and impact pressure after the same distance of travelling. This can be observed from the results at the first baffle in Fig. 8. As the density increases from $1828 \mathrm{~kg} / \mathrm{m}^{3}$ to $2259 \mathrm{~kg} / \mathrm{m}^{3}$, the peak pressure first grows and then drops when the density is beyond a threshold. Consequently, there should be a density which gives rise to the maximum impact pressure. Again, this trend cannot be clearly observed at other baffles due to the jet flow and complex flow dynamics.

\section{Discussion}

Reliable evaluation of impact force of debris flows on structures is very important for designing hazard mitigation infrastructures such as drainage channels and check dams. Generally, there are three categories of models to estimate the impact force of debris flow against structures: (1) Hydraulic models based on the fluid behaviour of the debris flow Hungr et al. (1984); Armanini and Scotton (1992); Hübl and Holzinger (2003); Canelli et al. (2012); Cui et al. (2015); (2) Fluid-solid body collision model considering both normal and tangential forces between flows and walls Jiang and Zhao (2015) and drag forces Vagnon and Segalini (2016); (3) shock wave collision model based on the shock wave speed inside a debris flow Wei (1996); Eglit et al. (2007). Usually, the last two models require particular input parameters such as the stiffness and strength of the impacting bodies and the shock wave speed. These parameters are extremely difficult to obtain for real world debris flow events. As a result, the hydraulic models become popular for calculating the impact force of debris flows as they only require the flow depth and flow velocity, which can be easily measured in real cases. Therefore, the results obtained from the SPH simulations are compared with predictions given by some popular hydraulic models.

In hydraulic models, the impact forces are proportional to either hydrostatic pressure or kinetic flow depth. Thus, the hydraulic models can be further grouped into hydrostatic, hydrodynamic, and mixed models. The hydrostatic model assumes that the impact pressure is proportional to the hydrostatic pressure exerted on fixed structures by debris materials. The model has the following form

$$
P_{\text {peak }}=k_{1} \rho g h
$$

The hydrostatic model is very simple yet popular because it only requires two parameters, i.e. the debris density $\rho$ and flow depth $h$. The empirical parameter $k_{1}$ ranges from 2.5 to 11 Armanini and Scotton (1992); Armanini (1997); Scotton and Deganutti (1997). The hydrostatic model does not consider the influence of the flow dynamics. Especially, the influence of flow velocity is not considered.

On the other hand, the hydrodynamic model is proposed by considering the variation in fluid dynamics.

$$
P_{\text {peak }}=k_{2} \rho u^{2}
$$

In literature Mizuyama (1979); Hungr et al. (1984); Zhang (1993); Bugnion et al. (2012); Canelli et al. (2012), the dynamic coefficient $k_{2}$ ranges from 0.4 to 5.0. 
Furthermore, some more complex formulations derived from the hydrodynamic relation are listed in Table 3. For example, in some models the Froude number $F r$ is used to normalize the impact force Hübl and Holzinger (2003); Zanuttigh and Lamberti (2006); Armanini et al. (2011); Cui et al. (2015), whereas other researchers consider the influence of grain size distribution (GSD) Yu (1992).

Table 3. Models of impact force derived from hydro-dynamic

\begin{tabular}{ccc}
\hline Author & Model & Feature \\
\hline Hübl and Holzinger Hübl and Holzinger (2003) & $P_{\text {peak }}=5 \rho u^{0.8}(g h)^{0.6}$ & Considering $F r$ \\
Zanuttigh and Lamberti Zanuttigh and Lamberti (2006) & $P_{\text {peak }}=K \frac{(1+\sqrt{2} F r)^{2}}{2} \rho g(h)^{2}$ & Considering $F r$ \\
Armanini et al. Armanini et al. (2011) & $P_{\text {peak }}=\left(1+0.5 F r^{2}\right)\left(1+\frac{\alpha F r^{2}}{1+0.5 F r^{2}}\right)$ & Considering $F r$ \\
Cui et al. Cui et al. (2015) & $P_{\text {peak }}=5.3 F r^{-1.5} \rho u^{2}$ & Considering $F r$ \\
Yu Yu (1992) & $P_{\text {peak }}=\sqrt{d_{\text {max }}} \rho u^{2} / g$ & Considering GSD \\
\hline
\end{tabular}

The mixed models consider both the hydrostatic and hydrodynamic effects Cascini et al. (2000); Arattano and Franzi (2003); Brighenti et al. (2013), which has the following general form

$$
P_{\text {peak }}=0.5 \rho g h+\rho u^{2}
$$

Based on the SPH simulation results, we can get the flow velocity, depth, and pressure at any specified position. And then, we use this information to calculate the impact stress by substituting them into the empirical equations. Here, the peak pressures obtained from the SPH simulations at 1\# baffle are compared with the predictions obtained using the empirical models. The employed models are the hydrostatic and hydrodynamic models Eq. (13) and (14), the Hübl and Holzinger model Hübl and Holzinger (2003), the Zanuttigh and Lamberti model Zanuttigh and Lamberti (2006), the Armanini model Armanini et al. (2011), and the Cui model Cui et al. (2015). The ranges of predicted impact force are $4.5-15.5 \mathrm{kPa}, 4.2-23.2 \mathrm{kPa}, 9-38.8 \mathrm{kPa}$, and 13.5 - $206.6 \mathrm{kP}$ from the Zanuttigh and Lamberti model, the hydrostatic model, the Armanini model, and the hydrodynamic model, respectively. As these models do not consider the inclination of baffle surface, they should be compared with the numerical results with square baffles. It is found that generally, the Zanuttigh and Lamberti model gives impact pressure lower than the numerical results, while the numerical results are close to the upper limits of the hydrostatic and Armanini models. The impact pressure given by the hydrodynamic model has a wide range, covering the numerical results. Although the Hübl and Holzinger model does not give a range of impact pressure, its prediction agrees very well with the numerical results with square baffles. However, it cannot be used to predict the impact pressure at triangle and trapezoid baffles, as it does not consider the influence of the inclination of baffle surface. Furthermore, the Cui model gives impact pressures very close to the upper limit of the hydrostatic model but lower than the numerical results. In addition, most of the models reasonably predict the decrease of impact pressure after the density is larger than a threshold.

In summary, some of the models, e.g. the Armanini model and the Hübl and Holzinger model, give pressure prediction quite close to the numerical results obtained in the channel with square baffles. However, the influence of the inclination of baffle surface is not considered in these empirical models. Thus, they cannot be applied to structures with inclined surface.

Based on the empirical models discussed above, it can be seen that the density and velocity are the dominant factors affecting the impact force. Furthermore, debris flows are gravity-driven flow and the impact forces are mainly determined by the kinetic energy. Therefore, a dimensionless variable representing the ratio between kinetic energy and potential energy, the Froude number Fr, is introduced to quantify the flow state Hübl and Holzinger (2003); Cui et al. (2015). The $F r$ governs the dynamics of channelized debris flows. It is the ratio between inertial and gravitational forces, namely, $F r=u /(g h \cos \theta)^{2}$. The parameter $F r$ also can be used to quantify the scale free relationships Hübl et al. (2009); Iverson (2015) between the small-scale laboratory modelling and full-scale field events. In addition, the angle between flowing direction and the impact face of structure (i.e. the inclined angle of baffle surface) also has a remarkable influence on the impact force Shieh et al. (2008). Considering these factors, a simple expression to estimate the impact force of debris flow on the structures given below

$$
P_{\text {peak }}=4.2 F r^{-1.2} \lambda \rho(u \sin \beta)^{2}
$$




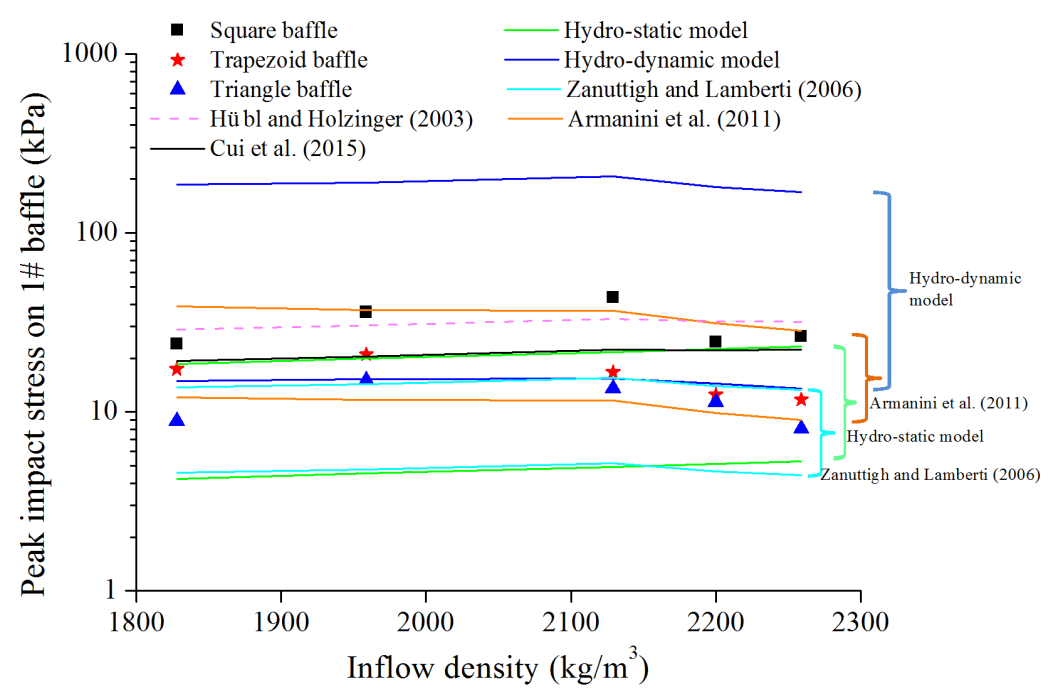

Figure 9: The peak impact stress on 1\# baffle obtained in this study and the results from previous formulas.

where $\beta$ is the angle between the direction normal to the face of structure and the flow direction, $\lambda$ is a regularization coefficient that considers the effect of density on the impact force, which can be expressed as

$$
\lambda=c_{1}\left(\frac{\rho}{\rho_{\max }}-1\right)^{2}+c_{2}
$$

where $\rho_{\max }$ is the threshold density. As discussed in Section 5.2, the impact force is expected to reach a maximum value at a threshold density $\rho_{\max }$. According to the numerical results, for the debris material used in this study, the threshold density is estimated as $\rho_{\max }=2000 \mathrm{~kg} / \mathrm{m}^{3}$. By fitting the numerical results, the constants $c_{1}$ and $c_{2}$ are taken as -50.0 and 1.3 , respectively. Note that the parameters $\rho_{\max }, c_{1}$, and $c_{2}$ may have different values for other type of debris materials.

The comparison between the numerical results and the prediction by the proposed model Eq. 16 is given in Fig. 10 . Overall, the proposed equation gives predictions well corroborated by the numerical results. It captures the influence of density on the impact pressure. More importantly, it considers the inclination of the baffle surface. The model predictions are in good agreement with numerical results from channels with different baffle shapes. The comparison preliminarily shows that the presented relation is applicable for estimating impact force on different shape of baffles.

More comparisons with laboratory test results and field data are necessary to check the capability of the proposed model. As an example of the investigation, the regression coefficient $\alpha$ recommended by Hübl et al. Hübl et al. (2009) that derived from the Eq. (16) is defined as following:

$$
\alpha=\frac{P_{\text {peak }}}{\lambda \rho(u \sin \beta)^{2}}=4.2 \mathrm{Fr}^{-1.2}
$$

The deduced expression of $\alpha$ is compared with the experimental data and field monitoring results of other researchers Zhang and Yuan (1985); Hübl and Holzinger (2003); Tiberghien et al. (2007); Proske et al. (2011); Cui et al. (2015); $\mathrm{Ng}$ et al. (2016) in Fig. 11. It can be seen that the expression of regression coefficient $\alpha$ is in qualitative agreement with the data, indicating the robustness and extrapolation capability of the proposed model.

Additionally, it should be kept in mind that single-phase homogeneous flow is assumed in the SPH simulation, meaning that the influence of coarse-grained materials is neglected. Coarse-grained materials like gravels and boulders moving as suspended load or bed load in debris flows have a significantly influence on the impact forces. It is expected that the impact pressure induced by boulders on a structure is much larger (two to three orders of magnitude He et al. (2016)) than the dynamic pressure of the slurry due to the high stiffness of the boulder and short interaction time Scheidl et al. (2013); Cui et al. (2015); He et al. (2016); Song et al. (2018). Since the time-force-functions for 


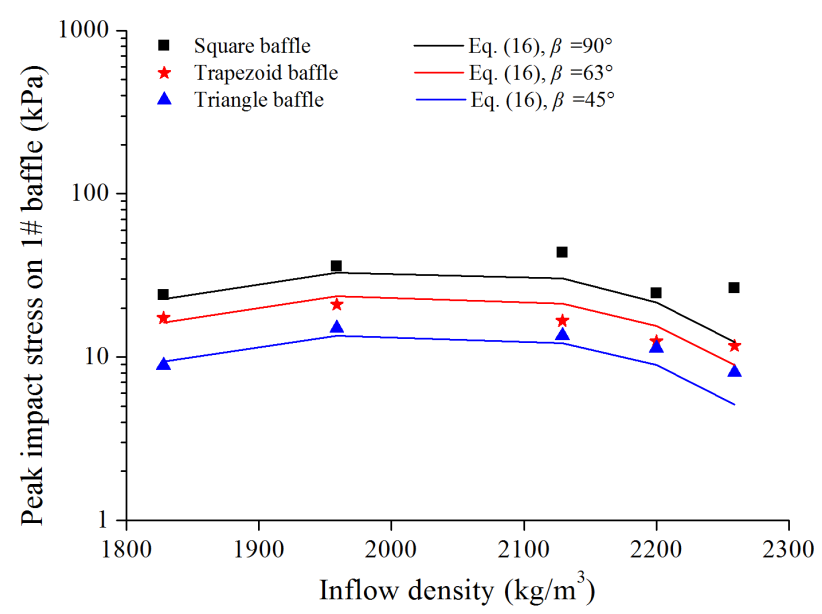

Figure 10: Comparison of the calculated results by Eq. (16) and numerical simulation data.

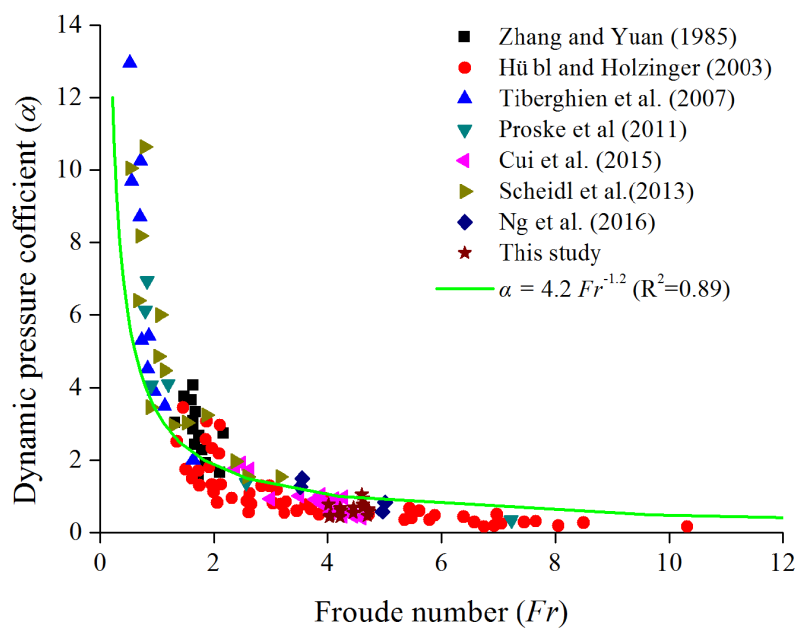

Figure 11: Relationship between empirical coefficient $\alpha$ and Froude number $F r$.

debris flow, as well as the interaction between fluid and solid phases in debris materials are still unclear, a systematic investigation of the influence of coarse-grained materials is required. It is possible to model debris flows with coarsegrained materials with coupled SPH and discrete element method (SPH-DEM). This, however, is out of the scope of this study.

\section{Conclusions}

In this study, the smoothed particle hydrodynamics is adopted to study the influence of baffle shapes on impact force between debris flow and step-pool structures in debris drainage channels. Three baffle shapes, e.g. square $\left(90^{\circ}\right)$, trapezoid $\left(63^{\circ}\right)$, and triangle $\left(45^{\circ}\right)$, are considered in the investigation. The debris material is modelled as weaklycompressible non-Newtonian fluid using the regularized Bingham model. Two drainage channel tests with square and triangle baffles are performed to validate the numerical model. A series of numerical simulations with varying baffle shape and material density are carried out. Based on the numerical results and their comparison with empirical relations in literarure, the following conclusions can be drawn:

(1) The flow dynamics in the step-pool system is very complex due to the jet flow induced by the violent impact between the flow and the first baffle. This complex flow condition makes the analysis of impact force on other baffles 
and the overall system resistance difficult. Nevertheless, we find that there is a weak dependence between the overall resistance and the inclination of baffle surface. A larger inclination usually results in slower flow and higher energy dissipation.

(2) The impact forces at the first baffle are more regularly dependent on baffle shape and material density. A larger inclination leads to higher impact pressure. However, in the step-pool system, the largest pressure measured at subsequent baffles is usually much larger than that at the first baffle due to the jet flow. Therefore, the impact pressure obtained from empirical models can only be applied to the first baffle.

(3) The influences of material density are twofold. With higher density the kinetic energy before the impact is larger; however, materials with high density usually have less fluidity due to high viscosity, which leads to less kinetic energy. There exists a threshold density which gives rise to the maximum impact pressure. Again, this conclusion only applies to the first baffle.

(4) Based on the numerical results, an empirical relation considering the influence of baffle shape is proposed to better compute the impact pressure. Comparison between the model prediction and data from literature show its applicability. This formulation might be used to guide the design of debris-resisting barriers with inclined surface.

\section{ACKNOWLEDGMENT}

The authors would like to thank the funding Supported by the Strategic Priority Research Program of the Chinese Academy of Sciences (grant No. XDA23090403), the Sichuan Science and Technology Program, the Youth Innovation Promotion Association of the CAS (Grant No. 2017426), the State Scholarship Fund (201804910306) of China Scholarship Council, the CAS "Light of West China" Program. The work is also partially funded by the National Natural Science Foundation of China (grant No. 51709230), H2020 Marie Skłodowska-Curie actions 693 RISE 2017 HERCULES (grant No. 778360 ), and FRAMED (grant No. 734485)

\section{References}

Stefan Adami, Xiangyu Y Hu, and Nikolaus A Adams. A generalized wall boundary condition for smoothed particle hydrodynamics. Journal of Computational Physics, 231(21):7057-7075, 2012.

M Arattano and LJNH Franzi. On the evaluation of debris flows dynamics by means of mathematical models. Natural Hazards and Earth System Science, 3(6):539-544, 2003.

A Armanini and P Scotton. Experimental analysis on the dynamic impact of a debris flow on structures. Internationales symposion interpraevent, 6:107-116, 1992.

A Armanini, M Larcher, and M Odorizzi. Dynamic impact of a debris flow front against a vertical wall. In R Genevois and L Douglas, editors, 5th International Conference on Debris-flow Hazard Mitigation, pages 1041-1049, Casa Editrice Universita La Spaienza: Roma, 2011.

Aronne Armanini. On the dynamic impact of debris flows. In Recent developments on debris flows, pages 208-226. Springer, 1997.

Roberto Brighenti, Andrea Segalini, and Anna Maria Ferrero. Debris flow hazard mitigation: a simplified analytical model for the design of flexible barriers. Computers and Geotechnics, 54:1-15, 2013.

Louis Bugnion, Brian W McArdell, Perry Bartelt, and Corinna Wendeler. Measurements of hillslope debris flow impact pressure on obstacles. Landslides, 9(2):179-187, 2012.

Francesco Calvetti, Claudio di Prisco, Irene Redaelli, Anna Sganzerla, and Emmanouil Vairaktaris. Mechanical interpretation of dry granular masses impacting on rigid obstacles. Acta Geotechnica, pages 1-17, 2019.

L Canelli, Anna Maria Ferrero, M Migliazza, and A Segalini. Debris flow risk mitigation by the means of rigid and flexible barriers-experimental tests and impact analysis. 2012.

L Cascini, D Guida, N Nocera, G Romanzi, and G Sorbino. A preliminary model for the landslides of may 1998 in campania region. In Proc 2nd Int. Symposium on Geotechnics of Hard Soil-Soft Rock, volume 3, pages 1623-1649. Balkema Napoli, 2000.

Guillaume Chambon, R Bouvarel, D Laigle, and Mohamed Naaim. Numerical simulations of granular free-surface flows using smoothed particle hydrodynamics. Journal of Non-Newtonian Fluid Mechanics, 166(12-13):698-712, 2011.

Jian-Yu Chen, Fue-Sang Lien, Chong Peng, and Eugene Yee. Gpu-accelerated smoothed particle hydrodynamics modeling of granular flow. Powder Technology, 359:94-106, 2020.

Jiangang Chen, Xiaoqing Chen, Yun Li, and Fei Wang. An experimental study of dilute debris flow characteristics in a drainage channel with an energy dissipation structure. Engineering geology, 193:224-230, 2015.

Jiangang Chen, Xiaoqing Chen, Wanyu Zhao, and Yong You. Debris flow drainage channel with energy dissipation structures: experimental study and engineering application. Journal of Hydraulic Engineering, 144(10):06018012, 2018.

XQ Chen, DJ Li, and P Cui. Calculating buried depth of transverse sills for the debris flow drainage groove with soft foundation. Journal of HeFei University of Technology, 32(10):1590-1593, 2009.

Anne Chin. The morphologic structure of step-pools in mountain streams. Geomorphology, 27(3-4):191-204, 1999.

CE Choi, Y Cui, LHD Liu, CWW Ng, and SDN Lourenço. Impact mechanisms of granular flow against curved barriers. Géotechnique Letters, 7 (4):330-338, 2017. 
Alejandro JC Crespo, José M Domínguez, Benedict D Rogers, Moncho Gómez-Gesteira, S Longshaw, RJFB Canelas, Renato Vacondio, A Barreiro, and O García-Feal. Dualsphysics: Open-source parallel cfd solver based on smoothed particle hydrodynamics (sph). Computer Physics Communications, 187:204-216, 2015.

P Cui, C Zeng, and Y Lei. Experimental analysis on the impact force of viscous debris flow. Earth Surface Processes and Landforms, 40(12): $1644-1655,2015$.

Zili Dai, Yu Huang, Hualin Cheng, and Qiang Xu. 3d numerical modeling using smoothed particle hydrodynamics of flow-like landslide propagation triggered by the 2008 wenchuan earthquake. Engineering Geology, 180:21-33, 2014.

Zili Dai, Yu Huang, Hualin Cheng, and Qiang Xu. Sph model for fluid-structure interaction and its application to debris flow impact estimation. Landslides, 14(3):917-928, 2017.

ME Eglit, VS Kulibaba, and M Naaim. Impact of a snow avalanche against an obstacle. formation of shock waves. Cold Regions Science and Technology, 50(1-3):86-96, 2007.

XJ Fei and AP Shu. Movement mechanism and disaster control for debris flow. Beijing, China: Press of Univ. Tsinghua, 2004.

Elliot James Fern and Kenichi Soga. The role of constitutive models in mpm simulations of granular column collapses. Acta Geotechnica, 11(3): 659-678, 2016

Siming He, Wei Liu, and Xinpo Li. Prediction of impact force of debris flows based on distribution and size of particles. Environmental Earth Sciences, 75(4):298, 2016

Alexis Hérault, Giuseppe Bilotta, Annamaria Vicari, Eugenio Rustico, and Ciro Del Negro. Numerical simulation of lava flow using a gpu sph model. Annals of Geophysics, 54(5), 2011.

Kaiheng Hu, Fangqiang Wei, and Yong Li. Real-time measurement and preliminary analysis of debris-flow impact force at jiangjia ravine, china. Earth Surface Processes and Landforms, 36(9):1268-1278, 2011.

Hai Huang, DT Ma, and XL Wang. Experimental study on the relationship between the velocity of debris flow and structure of the dongchuan debris flow channel. Journal of Mountain Science, 27(5):551-556, 2009.

Yu Huang, Weijie Zhang, Qiang Xu, Pan Xie, and Liang Hao. Run-out analysis of flow-like landslides triggered by the ms 8.02008 wenchuan earthquake using smoothed particle hydrodynamics. Landslides, 9(2):275-283, 2012.

J Hübl and G Holzinger. Development of design basis for crest open structures for debris flow management in torrents: miniaturized tests for the efficiency estimation of debris flow breakers. WLS Report, 2003.

Johannes Hübl, Jürgen Suda, Dirk Proske, Roland Kaitna, and Christian Scheidl. Debris flow impact estimation. In Proceedings of the 11th international symposium on water management and hydraulic engineering, Ohrid, Macedonia, pages 1-5, 2009.

O Hungr, GC Morgan, and R Kellerhals. Quantitative analysis of debris torrent hazards for design of remedial measures. Canadian Geotechnical Journal, 21(4):663-677, 1984.

K Hutter, B Svendsen, and D Rickenmann. Debris flow modeling: A review. Continuum mechanics and thermodynamics, 8(1):1-35, 1994.

R M Iverson and R P Denlinger. Flow of variably fluidized granular masses across three-dimensional terrain: 1. coulomb mixture theory. Journal of Geophysical Research: Solid Earth, 106(B1):537-552, 2001.

Richard M Iverson. Scaling and design of landslide and debris-flow experiments. Geomorphology, 244:9-20, 2015.

YJ Jiang and Y Zhao. Experimental investigation of dry granular flow impact via both normal and tangential force measurements. Géotechnique Letters, 5(1):33-38, 2015.

A Larese, R Rossi, E Oñate, and S R Idelsohn. Validation of the particle finite element method (pfem) for simulation of free surface flows. Engineering Computations, 2008.

A Leonardi, GR Goodwin, and M Pirulli. The force exerted by granular flows on slit dams. Acta Geotechnica, pages 1-15, 2019.

DJ Li. Theory and practice about reducingdebris flow hazards, 1997.

ZH Li, YJ Jiang, Q Lv, LR Sousa, and MC He. Consistent modeling of a catastrophic flowslide at the shenzhen landfill using a hydro-elasto-plastic model with solid-fluid transition. Acta Geotechnica, 13(6):1451-1466, 2018.

Chuan Lin, Manuel Pastor, Angel Yague, Saeid Moussavi Tayyebi, Miguel Martin Stickle, Diego Manzanal, Tongchun Li, and Xiaoqing Liu. A depth-integrated sph model for debris floods: application to lo wai (hong kong) debris flood of august 2005. Géotechnique, pages 1-21, 2019.

SL Liu, Y You, JF Liu, HX Zhao, and H Sun. Experimental study of the erosion after debris flow drainage channel ribs. Journal of Lanzhzou university: natural sciences, 52(3):328-332, 2016.

X Lv, Q P Zou, and D Reeve. Numerical simulation of overflow at vertical weirs using a hybrid level set/VOF method. Advances in Water Resources, 34(10):1320-1334, 2011

Salvatore Marrone, MAGD Antuono, A Colagrossi, G Colicchio, D Le Touzé, and G Graziani. $\delta$-sph model for simulating violent impact flows. Computer Methods in Applied Mechanics and Engineering, 200(13-16):1526-1542, 2011.

AR Maxwell, AN Papanicolaou, RH Hotchkiss, ME Barber, and J Schafer. Step-pool morphology in high-gradient countersunk culverts. Transportation research record, 1743(1):49-56, 2001.

T Mizuyama. Computational method and some considerations on impulsive force of debris flow acting on sabo dams. Journal of the Japan Society of Erosion Control Engineering, 112:40-43, 1979.

Joe J Monaghan. Simulating free surface flows with sph. Journal of computational physics, 110(2):399-406, 1994.

Joseph J Monaghan. Sph without a tensile instability. Journal of Computational Physics, 159(2):290-311, 2000.

Alomir H Fávero Neto and Ronaldo I Borja. Continuum hydrodynamics of dry granular flows employing multiplicative elastoplasticity. Acta Geotechnica, 13(5):1027-1040, 2018.

CWW Ng, Dongri Song, CE Choi, LHD Liu, JSH Kwan, RCH Koo, and WK Pun. Impact mechanisms of granular and viscous flows on rigid and flexible barriers. Canadian Geotechnical Journal, 54(2):188-206, 2016.

M Pastor, A Yague, MM Stickle, Diego Manzanal, and P Mira. A two-phase sph model for debris flow propagation. International Journal for Numerical and Analytical Methods in Geomechanics, 42(3):418-448, 2018.

Chong Peng, Xiaogang Guo, Wei Wu, and Yongqi Wang. Unified modelling of granular media with smoothed particle hydrodynamics. Acta Geotechnica, 11(6):1231-1247, 2016.

Chong Peng, Shun Wang, Wei Wu, Hai-sui Yu, Chun Wang, and Jian-yu Chen. Loquat: an open-source gpu-accelerated sph solver for geotechnical 
modeling. Acta Geotechnica, pages 1-19, 2019.

Dirk Proske, Jürgen Suda, and Johannes Hübl. Debris flow impact estimation for breakers. Georisk, 5(2):143-155, 2011.

Christian Scheidl, Michael Chiari, Roland Kaitna, Matthias Müllegger, Alexander Krawtschuk, Thomas Zimmermann, and Dirk Proske. Analysing debris-flow impact models, based on a small scale modelling approach. Surveys in Geophysics, 34(1):121-140, 2013.

Paolo Scotton and Andrea M Deganutti. Phreatic line and dynamic impact in laboratory debris flow experiments. In Debris-Flow Hazards Mitigation: Mechanics, Prediction, and Assessment, pages 777-786. ASCE, 1997.

Chjeng-Lun Shieh, TING Chia-Hsien, and PAN Hung-Wen. Impulsive force of debris flow on a curved dam. International journal of sediment research, 23(2):149-158, 2008.

D Song, CE Choi, CWW Ng, and GGD Zhou. Geophysical flows impacting a flexible barrier: effects of solid-fluid interaction. Landslides, 15(1): 99-110, 2018.

Kamil Szewc. Smoothed particle hydrodynamics modeling of granular column collapse. Granular Matter, 19(1):3, 2017.

D Tiberghien, D Laigle, M Naaim, E Thibert, and F Ousset. Experimental investigations of interaction between mudflow and an obstacle. Debrisflow hazards mitigation: mechanics, prediction and assessment, Millpress, Rotterdam, 2007.

Federico Vagnon and Andrea Segalini. Debris flow impact estimation on a rigid barrier. Natural Hazards and Earth System Sciences, 16(7): 1691-1697, 2016.

Wei Wang, Guangqi Chen, Zheng Han, Suhua Zhou, Hong Zhang, and Peideng Jing. 3d numerical simulation of debris-flow motion using sph method incorporating non-newtonian fluid behavior. Natural Hazards, 81(3):1981-1998, 2016.

Zhao Yin Wang, Lijian Qi, and Xuzhao Wang. A prototype experiment of debris flow control with energy dissipation structures. Natural Hazards, 60(3):971-989, 2012.

Kevin A Waters and Joanna Crowe Curran. Investigating step-pool sequence stability. Water Resources Research, 48(7), 2012.

Hong Wei. Experimental study on impact force of debris flow heads. China Academy of Railway Sciences, 17(3):50-62, 1996.

C Wendeler, A Volkwein, A Roth, M Denk, and S Wartmann. Field measurements and numerical modelling of flexible debris flow barriers. Debris-Flow Hazards Mitig. Mech. Predict. Assess. Millpress, Rotterdam, pages 681-687, 2007.

Holger Wendland. Piecewise polynomial, positive definite and compactly supported radial functions of minimal degree. Advances in computational Mathematics, 4(1):389-396, 1995

Andrew C Wilcox, Ellen E Wohl, Francesco Comiti, and Luca Mao. Hydraulics, morphology, and energy dissipation in an alpine step-pool channel. Water Resources Research, 47(7), 2011.

Xilin Xia and Qiuhua Liang. A gpu-accelerated smoothed particle hydrodynamics (sph) model for the shallow water equations. Environmental modelling $\mathcal{E}$ software, 75:28-43, 2016.

Xiaoyang Xu, Jie Ouyang, Binxin Yang, and Zhijun Liu. Sph simulations of three-dimensional non-newtonian free surface flows. Computer Methods in Applied Mechanics and Engineering, 256:101-116, 2013.

FC Yu. A study on the impact force of debris-flow. Proceedings of the National Science Council Part A, Physical Science and Engineering, Taipei, Taiwan, 16(1):32-39, 1992.

G Yu, Z Wang, X Duan, and K Zhang. Artificial step-pool system for ecological restoration of a debris-flow ravine. In PROCEEDINGS OF THE CONGRESS-INTERNATIONAL ASSOCIATION FOR HYDRAULIC RESEARCH, number 1, page 291, 2007.

Barbara Zanuttigh and Alberto Lamberti. Experimental analysis of the impact of dry avalanches on structures and implication for debris flows. Journal of Hydraulic research, 44(4):522-534, 2006.

Ling Zhan, Chong Peng, Bingyin Zhang, and Wei Wu. A stabilized tl-wc sph approach with gpu acceleration for three-dimensional fluid-structure interaction. Journal of Fluids and Structures, 86:329-353, 2019.

Shucheng Zhang. A comprehensive approach to the observation and prevention of debris flows in china. Natural Hazards, 7(1):1-23, 1993.

Shucheng Zhang and Jianmo Yuan. Impact force of debris flow and its detection. Memoirs of Lanzhou Institute of Glaciology and Cryopedology, Chinese Academy of Sciences. Beijing: Science Press, pages 269-274, 1985.

H Zhu, YD Kim, and D De Kee. Non-newtonian fluids with a yield stress. Journal of Non-Newtonian Fluid Mechanics, 129(3):177-181, 2005. 